\title{
AQUATIC PLANT DIVERSITY OF FIVE LAKES IN NAGZIRANAVEGAON (NN) CORRIDOR, GONDIA DISTRICT OF MAHARASHTRA STATE, INDIA
}

\section{Mahendra Bhojram Raut ${ }^{1}$, Chintaman J. Khune ${ }^{2}$, Laxman P. Nagpurkar ${ }^{3}$}

1. Research Scholar, Dept. of Zoology, M. B. Patel College, Sakoli, Dist- Bhandara, Email2. Asso. Prof.\& Head, P. G. Dept. of Zoology, M. B. Patel College, Sakoli, Dist- Bhandara, Email3. Email-Asso. Prof., Dept. of Chemistry, M. B. Patel College, Sakoli, Dist- Bhandara, mahendraraut5@gmail.com, cjkhune@yahoo.comand 1p.nagpurkar@gmail.com

\begin{abstract}
:
Nagzira Navegaon(NN) corridor covers an area of about $620 \mathrm{~km}$ distributed in three Tahsil viz. Sadak Arjuni, Deori and Goregaon in Gondia district of Maharashtra state. The corridor was studied into five blocks and wetlands were selected from each blocks; Putli lake from Ghat section block, Naktya lake from Alebedar block, Umarzari lake from Jambhadi block, Rengepar lake from Sasekaran hills block and Chulbandh lake from Mundipar block. The present work was carried out from October 2014 to September 2016, it showed 70 species of aquatic plants belonging to 34 families. The dominant families were Asteraceae with 8 species followed by Poaceae with 6 species and 4 species each of Alismataceae and Lamiaceae. Based on the existence of aquatic plants in lake, 41 species were peripheral, 14 species were shore, 11 species were floating and 4 species were categorized as submerged. As per the IUCN status, 54 species were not assessed by IUCN and 16 species were under the least concern status out of the 70 species observed during the study period. The present study mainly deals with the identification, documentation and conservation needs of aquatic plants species in lakes of NN corridor.
\end{abstract}

Key words: - Aquatic plants, Diversity, Corridor, Conservation

\section{INTRODUCTION:}

Wetland biodiversity provides a variety of environmental services from its species that are essential at the global, regional and local level (Rao et. al., 2017). A wetland ecosystem is a complex independent system of plants, animals and microorganisms along with physical environment in which they live (Bhagyaleena et. al., 2012). Wetland are highly valuable, yet the most threatened ecosystems (Murphy et. al., 2003).The available freshwater is not evenly distributed throughout either at local or global level. It now rests on man to properly manage this ecosystem (Ukaet. al., 2009).Biological science has attempted to classify living organism and categorized the variability in nature for over a century. This has led to an understanding of its organization into communication about the plants and animals. Planning for environmentally sound and biodiversity friendly development has in recent years emerged as an important concern. Minimizing the loss of biodiversity is one of the key objectives in planning for such sustainable development (Pramod et al., 1997).Wetlands are capable of performing various functions as a result of physical, chemical and biological processes (Ramachandra et al.,2002). Wetlands have been shown to improve water quality by filtering out fertilizers and pesticides. There are around 7084 wetlands recorded in Gondia distict of which 66 wetlands under the jurisdiction of Irrigation department, 1266 wetlands under the jurisdiction of Zilla Parisad and 5752 others (Laxman et al., 2015).The corridor between Nagzira Wildlife Sanctuary and Navegaon National Park situated in Gondia district of Maharashtra provides an excellent habitat for avifauna in the form of water bodies with marshy plant growth, terrestrial 
platforms having thick as well as scattered trees and bushy vegetation (Bahuguna et al., 2010).Wild animals widely use this corridor for their movement between Nagzira and Navegaon. The wetlands situated in the corridor fulfill the water requirement of wild animals during their movement and lean season. NN corridor has number of wetlands and other pocket of water bodies distributed in the whole study area, but water bodies have been decreasing continuously in the corridor. 182.76 sq. $\mathrm{km}$. area in 1990, under wetlands in the corridor was reduced to $137.62 \mathrm{sq} . \mathrm{km}$. in 1999 ; and to 104.35 sq. km. in 2009 (Yadav et al., 2012). Aquatic plants maintain the habitat of various wetland dependent species, documentation of these wetland plant species is at the utmost importance.Therefore, the present study was undertaken to document the aquatic biodiversity of NN corridor.

\section{MATERIAL AND METHODS:-}

The investigation on wetland plants of the corridor was carried out in all three different seasons; winter (October to January), summer (February to May) and monsoon (June to September) from October 2014 to September 2016. Observed plants were photographed and plant specimens were identified as per Ugemuge (1986), Kodarkar, (1992), Cook(1996) and Fasset (2000).The aquatic plants were categorized on the basis of their existence in lake as submerged, floating, shore plants and peripheral plants. Conservation needs of the aquatic plants were assessed on the basis of IUCN red list status.

\section{RESULT AND DISCUSSION}

In the present investigation, 70 species of aquatic plants belonging to 34 families were observed from 5 wetlands viz. Putli, Naktya, Umarzari, Rengepar and Chulbandh lake of NN corridor. Among the 34 families the Asteraceae were reported 8 species followed by Poaceae with 6 species and 4 species each of Amaranthaceae and Lamiaceae, 3 species each of Cyperaceae, Euphorbiaceae, Fabaceae, Hydrocharitaceae, Malvaceae, Convolvulaceae and
Solanaceae, 2 species each of Acanthaceae, Alismataceae,Nymphaceae and Onagraceae and a single species was reported from family Apocynaceae, Aponogetonaceae, Araceae, Arecaceae, Boraginaceae, Capparaceae, Ceratophyllaceae, Characeae, Commelinaceae, Meliaceae, Menyanthaceae, Nelumbonaceae, Oxalidaceae, Papaveraceae, Polygonaceae, Phyllanthaceae, Trapaceae, Typhaceae and Verbenaceae. In the present study majority of the aquatic plants $77 \%$ were not assessed followed by $23 \%$ aquatic plants were under least concern as per the IUCN red list status observed in $\mathrm{NN}$ corridor. Alien invasive plants pose a major threat to wetland species. In the present investigation aquatic invaders such as Ipomoea carnea (Beshram) was observed at the peripheral area at all 5 lakes viz. Putli, Naktya, Umarzari, Rengepar and Chulbandh lake

Aquatic plants with 16 number of species in NN corridor has been classed as Least Concern (LC) and 54 number of species were data deficientas per the IUCN red list status out of the 70 species observed in NN corridor. The number of plant species assessed as data deficient reflects the low level of knowledge of many wetland plants as it has not been assessed by IUCN. The species assessed here also represent only from five selected wetlands from NN corridor. Should an attempt have been made to assess all known aquatic plants (perennial and non-perennial) from all other lakes then the proportion of species assessed as data deficient would undoubtedly have been significantly higher than present investigation.The diversity and status of aquatic plants at 5 lakes during the present study was done to generate quantitative information about wetland plants diversity, the result shows the position of wetlands in corridor was not satisfactory as the aquatic plants are vanishes gradually and again invasive species invades the lake area. If present scenario of degradation of wetlands continue, the corridor existence would be severely threatened it can 
affects the wetland biodiversity and movement of wild animal through this corridor. The ninety villages located in the corridor and people are directly dependent on these wetland resources for fish, water for agriculture and others so it was felt that the success of reconnecting people with their ecosystem lies within the people themselves and their empowerment. They must feel a sense of ownership of conservation or restoration sites. All stakeholders like neighborhoods, Government (up to lowest level), and private agencies or NGO's must have participative approach in planning, implementation and evaluating of the restoration work as equals.

\section{ACKNOWLEDGMENTS}

We wish to express our sincere gratitude to Forest Department, Govt. of Maharashtra and local Biodiversity Committees (BMC's) for granting permission to undertake research on wetland plant diversity in NagziraNavegaon(NN) corridor. We aregrateful to all the volunteers and villagers for their support during the entire field work.

\section{REFERENCES}

Bahuguna A., Sati J. and Tak P. (2010): Sighting of Black Stork (Ciconianigra) in Kalesar National Park, Yamunanagar district, Haryana, India. Newsletter for Birdwatchers,48(4): 49-50.

Bhagyaleena P. and Gopalan R. (2012): Aquatic plant diversity of ponds in Nemmara panchayat, Palakkad district, Kerala, India. International Journal of Scientific and Research Publication, 2 (8).

Cook C. (1996): Aquatic and Wetland Plants in India. Oxford University Press, London.

Fasset (2000): A manual of aquatic plants, Agrobios (India), Jodhapur.

Kodarkar M. (1992): Methodology for water analysis, physico-chemical, biological and micro-biological India Association of Aquatic Biologists, Hydrabad, Journal of Aquatic Biology, 9 (1\&2): 30.
Laxman P. and Mahendra R. (2015): The sacred groves and conservation of biodiversity of Ngzira WLS-Navegaon NP Corridor in Gondia district of Maharashtra State, India, International Journal of Researches in Biosciences, Agriculture and Technology, 2 (7): 333-339.

Murphy K., Dickinson G., Thomas S., Bini L., Dick K., Greaves K., Kennedy M., Livingstone S., McFerran H., Milne J., Oldroyd J. and Wingfield R. (2003): Aquatic plant communities and predictors of diversity in a sub-tropical river floodplains: The upper Rio Parana, Brazil, Aquatic Botany, 77: 257-176.

Pramod P., Ranjit D., Joshi N. and Gadgil M. (1997):Evaluating bird communities of Western Ghats to plan for a biodiversity friendly development. Current Science, 73 (2): 156-162.

Ramachandra T. (2002): Status of wetlands in Bangalore. Technical Report, 86.

Rao P., Yadav A. and Shah R. (2017): A survey on biodiversity of J. M. Patel College, Bhandara, Maharashtra. EssenceInternational Journal for Environmental Rehabilitation and Conservation,VII (2): 29-33.

Ugemuge N. (1986): Flora of Nagpur district published by Shree Prakashan, 43 Nagpur.

Uka U., Mohammed H. and Ovie S. (2009): Current diversity of aquatic macrophytes in Nigerian freshwater ecosystems, Journal of Aquatic Science, 13 (2): 9-15.

Yadav P., Kapoor M. and Sarma K. (2012): Land use land cover mapping, change detection and conflict analysis of NagziraNavegaon Corridor, Central India using geospatial technology. 
Table 1- Diversity and status of aquatic plants during study period

\begin{tabular}{|c|c|c|c|c|c|c|c|c|c|c|}
\hline \multirow[b]{2}{*}{$\begin{array}{l}\text { Sr. } \\
\text { No. }\end{array}$} & \multirow[b]{2}{*}{ Local name } & \multirow[b]{2}{*}{ Scientific name } & \multirow[b]{2}{*}{ Family } & \multirow[b]{2}{*}{ 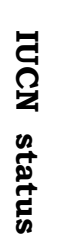 } & \multicolumn{6}{|c|}{ Name of lakes } \\
\hline & & & & & 总 & 园 & 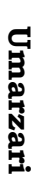 &  & 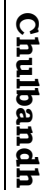 &  \\
\hline 1 & Talimkhan & Hygrophylaschulli & Acanthaceae & NA & & $\sqrt{ }$ & $\sqrt{ }$ & $\sqrt{ }$ & $\sqrt{ }$ & $\mathrm{P}$ \\
\hline 2 & Katekorsa & Asteracanthalongifolia & Acanthaceae & NA & & & $\sqrt{ }$ & $\sqrt{ }$ & $\sqrt{ }$ & $\mathrm{S}$ \\
\hline 3 & Jirapan & Sagittariatrifolia & Alismataceae & LC & $\sqrt{ }$ & $\sqrt{ }$ & $\sqrt{ }$ & $\sqrt{ }$ & & $\mathrm{S}$ \\
\hline 4 & Kadukand & Sagittariasagittifolia & Alismataceae & LC & & $\sqrt{ }$ & & $\sqrt{ }$ & $\sqrt{ }$ & $\mathrm{F}$ \\
\hline 5 & Bhaspatur & Alternatherasessilis & Amaranthaceae & NA & & $\sqrt{ }$ & $\sqrt{ }$ & $\sqrt{ }$ & $\sqrt{ }$ & $\mathrm{S}$ \\
\hline 6 & Chavli & Amaranthusblitum & Amaranthaceae & NA & $\sqrt{ }$ & $\sqrt{ }$ & $\sqrt{ }$ & $\sqrt{ }$ & $\sqrt{ }$ & $\mathrm{P}$ \\
\hline 7 & Kutri & Achyranthesaspera & Amaranthaceae & NA & $\sqrt{ }$ & $\sqrt{ }$ & $\sqrt{ }$ & $\sqrt{ }$ & $\sqrt{ }$ & $\mathrm{P}$ \\
\hline 8 & Silari & Celosia argentea & Amaranthaceae & NA & $\sqrt{ }$ & $\sqrt{ }$ & $\sqrt{ }$ & $\sqrt{ }$ & $\sqrt{ }$ & $\mathrm{P}$ \\
\hline 9 & Rui & Calatropisgigantea & Apocynaceae & NA & & $\sqrt{ }$ & $\sqrt{ }$ & $\sqrt{ }$ & $\sqrt{ }$ & $\mathrm{P}$ \\
\hline 10 & panz & Aponogetonnatans & Aponogetonaceae & LC & & $\sqrt{ }$ & $\sqrt{ }$ & $\sqrt{ }$ & & $\mathrm{F}$ \\
\hline 11 & Jalkumbha & Pistia stratiotes & Araceae & LC & $\sqrt{ }$ & $\sqrt{ }$ & & $\sqrt{ }$ & & $\mathrm{F}$ \\
\hline 12 & Shindi & Phoenix sylvestris & Arecaceae & NA & & $\sqrt{ }$ & & $\sqrt{ }$ & $\sqrt{ }$ & $\mathrm{P}$ \\
\hline 13 & PivliGodurli & Grangeamaderaspatena & Asteraceae & NA & & $\sqrt{ }$ & $\sqrt{ }$ & $\sqrt{ }$ & $\sqrt{ }$ & $\mathrm{P}$ \\
\hline 14 & Godurli & Sphaeranthusindicus & Asteraceae & LC & $\sqrt{ }$ & $\sqrt{ }$ & $\sqrt{ }$ & $\sqrt{ }$ & & $\mathrm{P}$ \\
\hline 15 & Kambarmodi & Tridaxprocumbens & Asteraceae & NA & $\sqrt{ }$ & $\sqrt{ }$ & $\sqrt{ }$ & $\sqrt{ }$ & $\sqrt{ }$ & $\mathrm{P}$ \\
\hline 16 & MajviLendurli & Xanthium strumarium & Asteraceae & NA & & $\sqrt{ }$ & & $\sqrt{ }$ & & $\mathrm{P}$ \\
\hline 17 & Pandhrasambhar & Vernoniacinerea & Asteraceae & NA & & $\sqrt{ }$ & $\sqrt{ }$ & $\sqrt{ }$ & & $\mathrm{P}$ \\
\hline 18 & Chatkachandani & Blumealacera & Asteraceae & NA & & $\sqrt{ }$ & $\sqrt{ }$ & $\sqrt{ }$ & $\sqrt{ }$ & $P$ \\
\hline 19 & Akkalkada & Acmella paniculata & Asteraceae & LC & $\sqrt{ }$ & $\sqrt{ }$ & $\sqrt{ }$ & $\sqrt{ }$ & $\sqrt{ }$ & $\mathrm{P}$ \\
\hline 20 & Dhondurli & Blumeaeriantha & Asteraceae & NA & $\sqrt{ }$ & & $\sqrt{ }$ & $\sqrt{ }$ & $\sqrt{ }$ & $\mathrm{P}$ \\
\hline 21 & Bhurundi & Heliotopiumindicum & Boraginaceae & NA & $\sqrt{ }$ & & $\sqrt{ }$ & $\sqrt{ }$ & $\sqrt{ }$ & $P$ \\
\hline 22 & Waghatichikna & Cappariszeylanica & Capparaceae & NA & & $\sqrt{ }$ & & $\sqrt{ }$ & & $\mathrm{P}$ \\
\hline 23 & $\begin{array}{l}\text { Vanarpushtyachi } \\
\text { la }\end{array}$ & $\begin{array}{c}\text { Ceratophyllumsubmersu } \\
\mathrm{m}\end{array}$ & Ceratophyllaceae & LC & & & & $\sqrt{ }$ & & $\mathrm{Sb}$ \\
\hline 24 & Gobryachila & Charaglobularis & Characeae & NA & & & $\sqrt{ }$ & $\sqrt{ }$ & & $\mathrm{Sb}$ \\
\hline 25 & Chipli & Merremiaemarginata & Convolvulaceae & LC & $\sqrt{ }$ & & $\sqrt{ }$ & $\sqrt{ }$ & $\sqrt{ }$ & $\mathrm{P}$ \\
\hline 26 & KarambuBhaji & Ipomoea aquatica & Convolvulaceae & LC & & $\sqrt{ }$ & & $\sqrt{ }$ & & $\mathrm{F}$ \\
\hline 27 & $\begin{array}{l}\text { Sadafuli/ } \\
\text { Beshram }\end{array}$ & Ipomoea carnea & Convolvulaceae & NA & $\sqrt{ }$ & $\sqrt{ }$ & $\sqrt{ }$ & & $\sqrt{ }$ & $\mathrm{P}$ \\
\hline 28 & Bhaskena & Cyanotis cristata & Commelinaceae & LC & $\sqrt{ }$ & $\sqrt{ }$ & $\sqrt{ }$ & $\sqrt{ }$ & $\sqrt{ }$ & S \\
\hline 29 & Pongli Gad & $\begin{array}{c}\text { Schoenoplectusarticulatu } \\
\text { s }\end{array}$ & Cyperaceae & NA & & $\sqrt{ }$ & & $\sqrt{ }$ & $\sqrt{ }$ & $\mathrm{S}$ \\
\hline 30 & Shikara & Kyllinga triceps & Cyperaceae & LC & & $\sqrt{ }$ & $\sqrt{ }$ & $\sqrt{ }$ & $\sqrt{ }$ & $\mathrm{S}$ \\
\hline
\end{tabular}


I J R B A T, Issue (VIII), Vol. I, Jan 2020: 123-128

\begin{tabular}{|c|c|c|c|c|c|c|c|c|c|c|}
\hline 31 & Nagarmotha & Cyperusrotundus & Cyperaceae & LC & $\sqrt{ }$ & $\sqrt{ }$ & $\sqrt{ }$ & $\sqrt{ }$ & $\sqrt{ }$ & $\mathrm{S}$ \\
\hline 32 & Botri & Chrozophorarottleri & Euphorbiaceae & NA & $\sqrt{ }$ & $\sqrt{ }$ & $\sqrt{ }$ & $\sqrt{ }$ & $\sqrt{ }$ & $\mathrm{P}$ \\
\hline 33 & Dudheli & Euphorbia hirta & Euphorbiaceae & NA & $\sqrt{ }$ & & $\sqrt{ }$ & $\sqrt{ }$ & $\sqrt{ }$ & $\mathrm{P}$ \\
\hline 34 & MothiLendurli & Ricinuscommunis & Euphorbiaceae & NA & $\sqrt{ }$ & $\sqrt{ }$ & & $\sqrt{ }$ & $\sqrt{ }$ & $\mathrm{P}$ \\
\hline 35 & Harduli & Crotolariaramosissima & Fabaceae & NA & $\sqrt{ }$ & $\sqrt{ }$ & $\sqrt{ }$ & $\sqrt{ }$ & & $\mathrm{S}$ \\
\hline 36 & Tarota & Cassia tora & Fabaceae & NA & $\sqrt{ }$ & $\sqrt{ }$ & $\sqrt{ }$ & $\sqrt{ }$ & $\sqrt{ }$ & $\mathrm{P}$ \\
\hline 37 & Palas & Butea monosperma & Fabaceae & NA & $\sqrt{ }$ & & $\sqrt{ }$ & $\sqrt{ }$ & $\sqrt{ }$ & $\mathrm{P}$ \\
\hline 38 & Chiul & Vallisneria spiralis & Hydrocharitaceae & NA & $\sqrt{ }$ & $\sqrt{ }$ & $\sqrt{ }$ & $\sqrt{ }$ & & $\mathrm{F}$ \\
\hline 39 & Katerichila & Najas minor & Hydrocharitaceae & NA & & $\sqrt{ }$ & & $\sqrt{ }$ & $\sqrt{ }$ & $\mathrm{F}$ \\
\hline 40 & Sakhryachila & Hydrillaverticillata & Hydrocharitaceae & $\mathrm{LC}$ & & $\sqrt{ }$ & $\sqrt{ }$ & $\sqrt{ }$ & $\sqrt{ }$ & $\mathrm{Sb}$ \\
\hline 41 & Astha & Ocimumamericanum & Lamiaceae & NA & $\sqrt{ }$ & $\sqrt{ }$ & $\sqrt{ }$ & $\sqrt{ }$ & $\sqrt{ }$ & $\mathrm{P}$ \\
\hline 42 & Tulsi & Ocimumtenuiflorum & Lamiaceae & NA & & $\sqrt{ }$ & $\sqrt{ }$ & $\sqrt{ }$ & & $\mathrm{P}$ \\
\hline 43 & KateriZandu & Leonotisnepetifolia & Lamiaceae & NA & $\sqrt{ }$ & & $\sqrt{ }$ & $\sqrt{ }$ & $\sqrt{ }$ & $\mathrm{P}$ \\
\hline 44 & Bhutganjya & Hyptissuaveolens & Lamiaceae & NA & $\sqrt{ }$ & & $\sqrt{ }$ & $\sqrt{ }$ & $\sqrt{ }$ & $\mathrm{P}$ \\
\hline 45 & Chikna & Common sida & Malvaceae & NA & $\sqrt{ }$ & $\sqrt{ }$ & $\sqrt{ }$ & $\sqrt{ }$ & $\sqrt{ }$ & $\mathrm{P}$ \\
\hline 46 & Dhendurli & Urenalobata & Malvaceae & NA & $\sqrt{ }$ & $\sqrt{ }$ & $\sqrt{ }$ & $\sqrt{ }$ & $\sqrt{ }$ & $\mathrm{P}$ \\
\hline 47 & Bhendi & Abelmoschusesculentus & Malvaceae & NA & $\sqrt{ }$ & & $\sqrt{ }$ & $\sqrt{ }$ & $\sqrt{ }$ & $\mathrm{P}$ \\
\hline 48 & Kadunimb & Azadirachtaindica & Meliaceae & NA & & & $\sqrt{ }$ & $\sqrt{ }$ & $\sqrt{ }$ & $\mathrm{P}$ \\
\hline 49 & Chaura & Nymphoideshydrophylla & Menyanthaceae & $\mathrm{LC}$ & & $\sqrt{ }$ & $\sqrt{ }$ & $\sqrt{ }$ & & $\mathrm{F}$ \\
\hline 50 & Powan & Nelumbonucifera & Nelumbonaceae & NA & & & $\sqrt{ }$ & & & $\mathrm{F}$ \\
\hline 51 & Kamal & Nymphaenouchali & Nymphaeaceae & NA & & & $\sqrt{ }$ & $\sqrt{ }$ & & $\mathrm{F}$ \\
\hline 52 & Kamalful & Nymphae pubescence & Nymphaeaceae & NA & & $\sqrt{ }$ & & $\sqrt{ }$ & & $\mathrm{F}$ \\
\hline 53 & Panlavang & Ludwigiaoctovalvis & Onagraceae & NA & & $\sqrt{ }$ & $\sqrt{ }$ & $\sqrt{ }$ & & $\mathrm{S}$ \\
\hline 54 & Rajoli & Ludwigiaadscendens & Onagraceae & NA & & $\sqrt{ }$ & $\sqrt{ }$ & $\sqrt{ }$ & $\sqrt{ }$ & $\mathrm{S}$ \\
\hline 55 & Tipani & Oxalis corniculata & Oxalidaceae & NA & $\sqrt{ }$ & & & $\sqrt{ }$ & $\sqrt{ }$ & $\mathrm{F}$ \\
\hline 56 & Utati & Argemonemexicana & Papaveraceae & NA & $\sqrt{ }$ & & $\sqrt{ }$ & $\sqrt{ }$ & $\sqrt{ }$ & $\mathrm{P}$ \\
\hline 57 & Devdhan & Oryzarufipogon & Poaceae & NA & & $\sqrt{ }$ & $\sqrt{ }$ & $\sqrt{ }$ & & $\mathrm{S}$ \\
\hline 58 & Ursudi & Vetiveriazizanioides & Poaceae & NA & & $\sqrt{ }$ & $\sqrt{ }$ & $\sqrt{ }$ & $\sqrt{ }$ & $\mathrm{S}$ \\
\hline 59 & Harari & Cynodondactylon & Poaceae & NA & $\sqrt{ }$ & $\sqrt{ }$ & $\sqrt{ }$ & $\sqrt{ }$ & & $\mathrm{S}$ \\
\hline 60 & Ghonyad & Themedatriandra & Poaceae & NA & $\sqrt{ }$ & & $\sqrt{ }$ & $\sqrt{ }$ & $\sqrt{ }$ & $\mathrm{P}$ \\
\hline 61 & Lahanasera & Aristidiasetaceae & Poaceae & NA & $\sqrt{ }$ & $\sqrt{ }$ & $\sqrt{ }$ & $\sqrt{ }$ & $\sqrt{ }$ & $\mathrm{P}$ \\
\hline 62 & Mothaasera & Aristidiaadscesionis & Poaceae & NA & $\sqrt{ }$ & & $\sqrt{ }$ & $\sqrt{ }$ & & $\mathrm{P}$ \\
\hline 63 & Siruli & Polygonumglabrum & Polygonaceae & $\mathrm{LC}$ & $\sqrt{ }$ & & $\sqrt{ }$ & $\sqrt{ }$ & $\sqrt{ }$ & $\mathrm{P}$ \\
\hline 64 & Bhuiamla & Phyllanthusniruri & Phyllanthaceae & NA & & & $\sqrt{ }$ & $\sqrt{ }$ & $\sqrt{ }$ & $\mathrm{P}$ \\
\hline 65 & KateriVanguli & Solanum surrattense & Solanaceae & NA & $\sqrt{ }$ & & $\sqrt{ }$ & $\sqrt{ }$ & $\sqrt{ }$ & $\mathrm{P}$ \\
\hline 66 & Phopondra & Physalis minima & Solanaceae & NA & & $\sqrt{ }$ & $\sqrt{ }$ & $\sqrt{ }$ & $\sqrt{ }$ & $\mathrm{P}$ \\
\hline 67 & Ranvanga & Solanum xanthocarpum & Solanaceae & NA & $\sqrt{ }$ & $\sqrt{ }$ & $\sqrt{ }$ & $\sqrt{ }$ & $\sqrt{ }$ & $\mathrm{S}$ \\
\hline 68 & Shingada & Trapanatans & Trapaceae & NA & & $\sqrt{ }$ & & & & $\mathrm{Sb}$ \\
\hline
\end{tabular}




\begin{tabular}{|c|c|c|c|c|c|c|c|c|c|c|}
\hline 69 & Kongasi & Typhaprovincialis & Typhaceae & NA & $\sqrt{ }$ & $\sqrt{ }$ & $\sqrt{ }$ & $\sqrt{ }$ & & $\mathrm{P}$ \\
\hline 70 & Ghaneri & Lantana camara & Verbenaceae & NA & $\sqrt{ }$ & & $\sqrt{ }$ & $\sqrt{ }$ & $\sqrt{ }$ & $\mathrm{P}$ \\
\hline \multicolumn{5}{|c|}{ Total } & $\begin{array}{l}3 \\
8\end{array}$ & $\begin{array}{l}4 \\
8\end{array}$ & $\begin{array}{l}5 \\
7\end{array}$ & $\begin{array}{l}6 \\
7\end{array}$ & $\begin{array}{l}4 \\
7\end{array}$ & \\
\hline
\end{tabular}

IUCN status:LC- Least Concern, NA- Not Assessed, Location status: P - Peripheral, S - Shore, F Floating, $\mathrm{Sb}-$ Submerged and $\sqrt{ }=$ Present

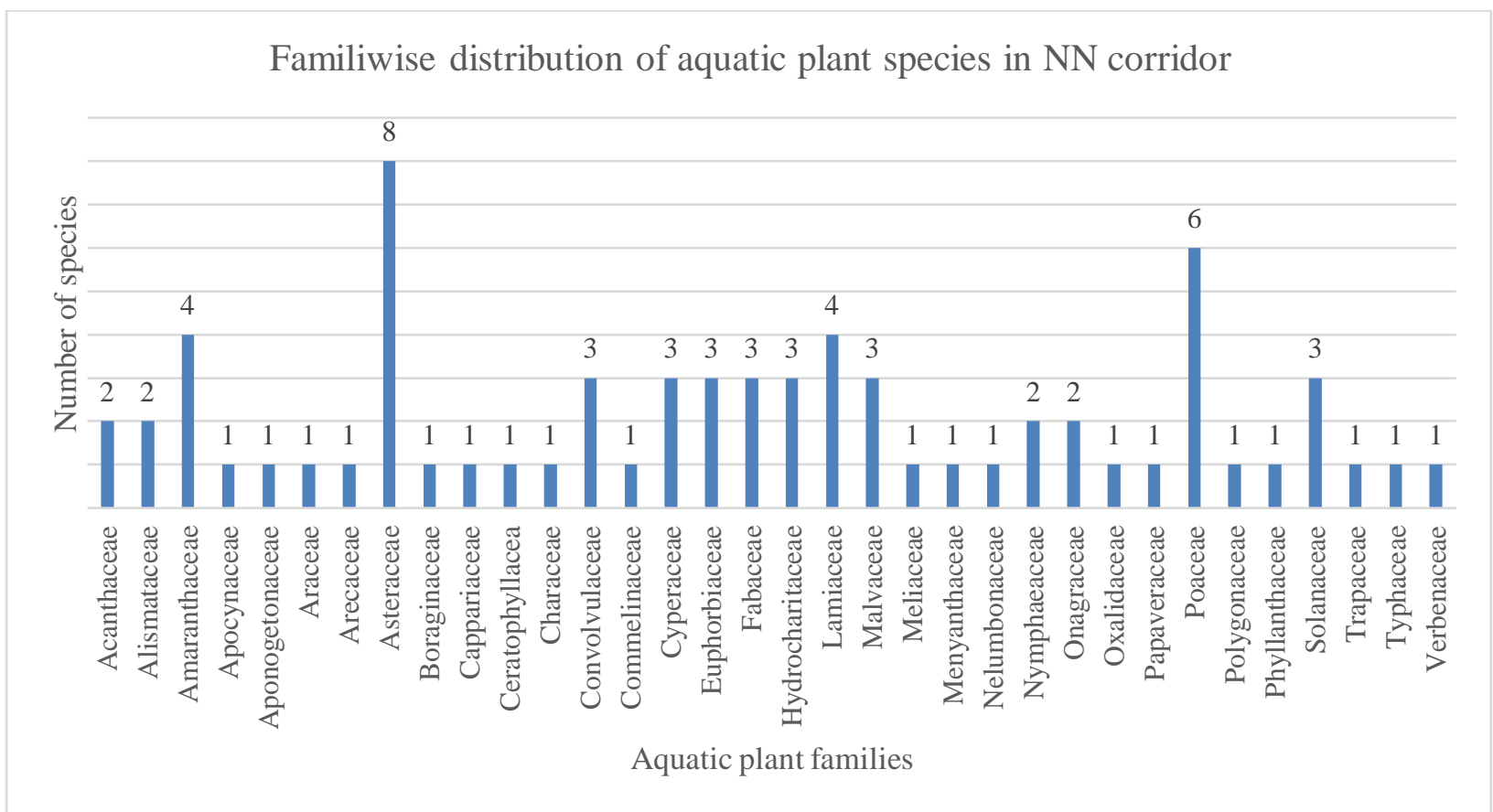

Figure 1- Number of aquatic plant species under different families in NN corridor

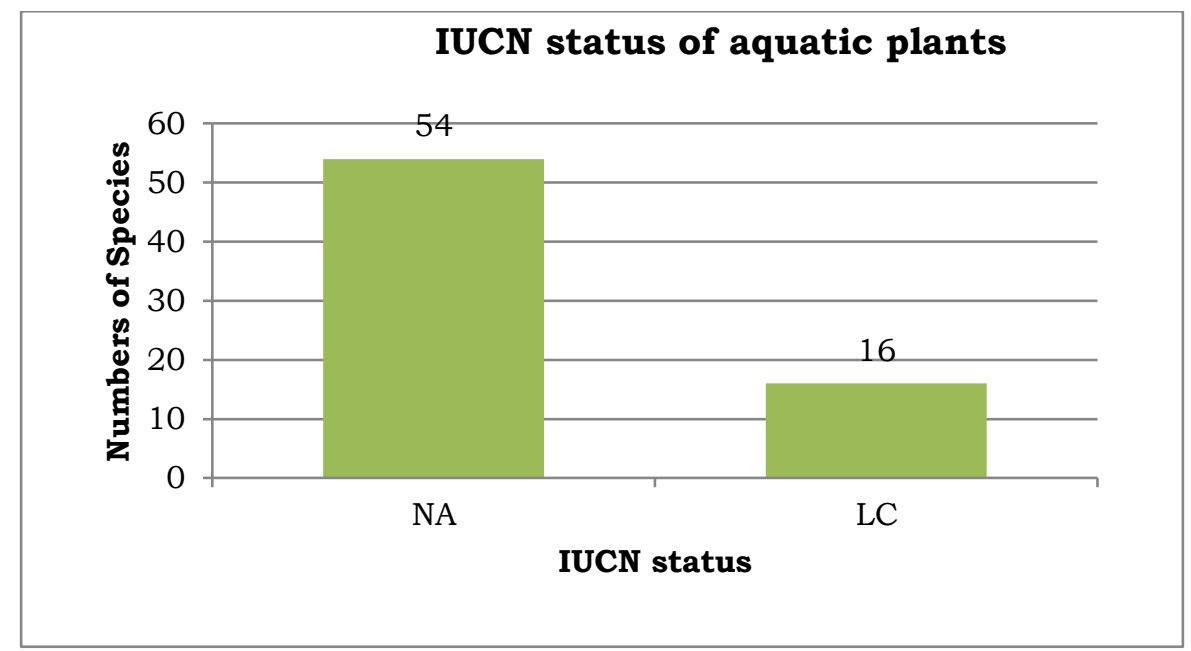

Figure 2- IUCN status of aquatic plants during study period 